\title{
Iliac access conduit facilitates endovascular aortic aneurysm repair and ipsilateral iliofemoral bypass
}

\author{
Hajime Kinoshita', Eiki Fujimoto', Hitoshi Sogabe², Hiroshi Fujita², \\ Taisuke Nakayama', Mikio Sugano', Hirotsugu Kurobe', Tamotsu Kanbara', \\ Takashi Kitaichi', and Tetsuya Kitagawa' \\ ${ }^{1}$ Department of Cardiovascular Surgery, Institute of Health Biosciences, the University of Tokushima \\ Graduate School, Tokushima, Japan, ${ }^{2}$ Imabari Daiichi Hospital, Ehime, Japan
}

\begin{abstract}
It may be difficult to access a route to deliver a stent-graft for abdominal aortic aneurysm in high-risk patients with bilateral iliofemoral occlusive disease. These two patients underwent both endovascular aortic aneurysm repair by a modified iliac access conduit technique and sequential ipsilateral iliofemoral artery bypass using the conduit, which provided excellent results. The iliac access conduit facilitates endovascular aortic aneurysm repair and ipsilateral iliofemoral bypass of high-risk patients with abdominal aortic aneurysm and bilateral iliofemoral occlusive disease. J. Med. Invest. 61 : 204-207, February, 2014
\end{abstract}

Keywords : iliac access conduit, bilateral iliofemoral occlusive diseases, endovascular aortic aneurysm repair

\section{INTRODUCTION}

A combination of Trans-Atlantic Intersociety Consensus D iliofemoral occlusive disease and a significant abdominal aortic aneurysm (AAA) is occasionally encountered. Previous studies have shown that approximately $40 \%-70 \%$ of patients with AAA were anatomically unsuitable for endovascular aortic aneurysm repair (EVAR) due to bilateral iliofemoral occlusive diseases (1). Extensive calcified atherosclerotic disease, occlusions, and tortuous and/ or narrow iliac arteries make the usual transfemoral access approach difficult. Two successful patients of both EVAR using the iliac access conduit as a safe endovascular arterial access and sequential iliofemoral artery bypass using the conduit are described in

Received for publication August 1, 2013 ; accepted August 19, 2013.

Address correspondence and reprint requests to Hajime Kinoshita MD, Department of Cardiovascular Surgery, Institute of Health Biosciences, the University of Tokushima Graduate School 318-15 Kuramoto, Tokushima 770-8503, Japan and Fax : +81-88633-7408. this report.

\section{CASE 1.}

A 77-year-old male exhibited both a huge infrarenal AAA and bilateral leg claudication. He had a history of cerebral infarction, hypertension and chronic kidney disease. Bilateral ankle-brachial pressure indexes (ABIs) were $0.75-0.80$. Preoperative computed tomo-angiography revealed long complete occlusion of the right external iliac artery and severe stenosis of the left external iliac artery (Figure 1 (a)).

\section{CASE 2.}

An 85-year-old male showed a significant infrarenal AAA and bilateral leg claudication. He had a history of chronic kidney disease and dementia. Preoperative bilateral ABIs were 0.71-0.72. Preoperative computed tomo-angiography revealed that the patient had complete occlusion of the left common iliac artery and severe stenosis of the right external iliac artery (Figure 1 (b)). 
(a)

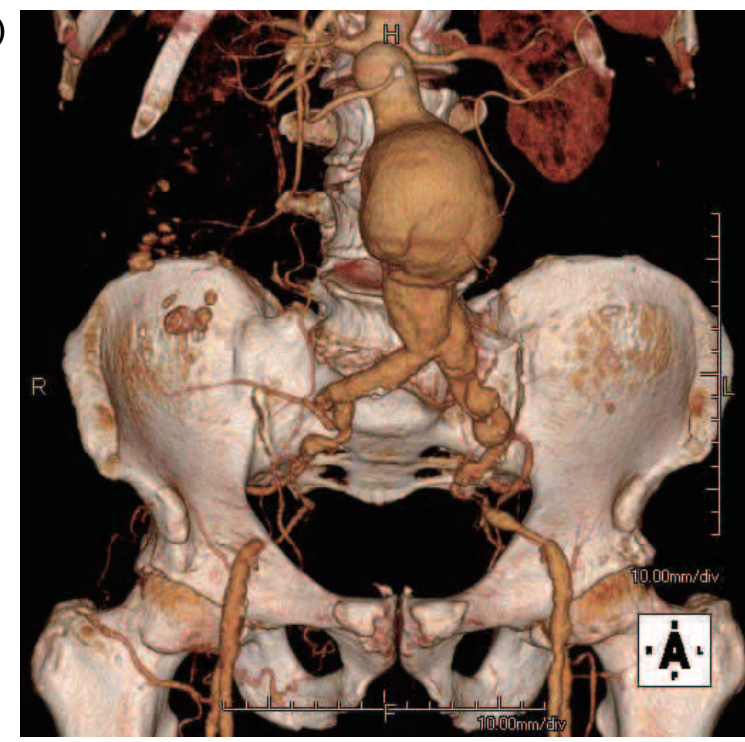

(b)

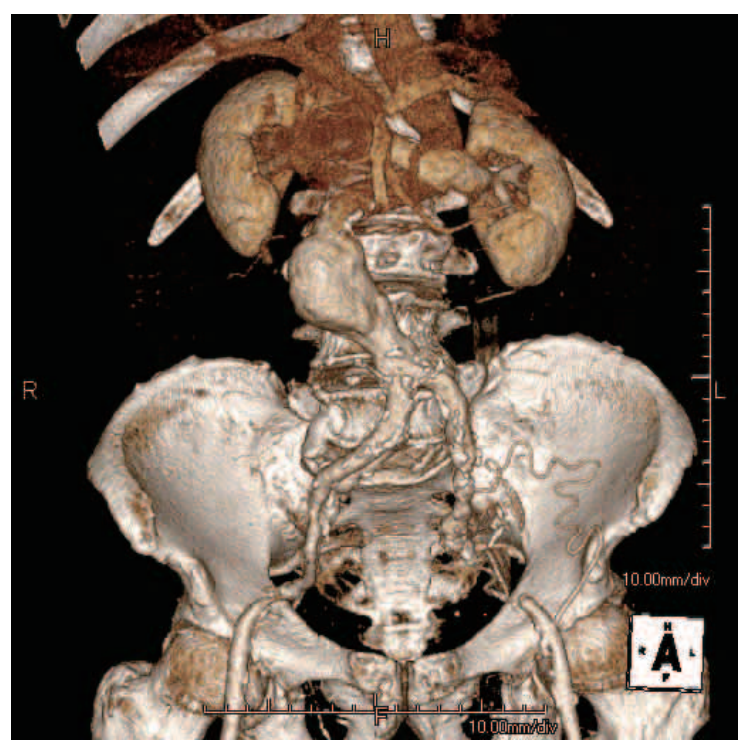

Figure 1. Preoperative 3D-computed tomo-angiography. Preoperative $3 \mathrm{D}$-computed tomo-angiography showed complete occlusion of the right external iliac artery and severe stenosis of the left external iliac artery in Case 1 (a) and complete occlusion of the left external iliac artery and severe stenosis of the right external iliac artery in Case 2 (b).

The patients were of advanced age with poor activities of daily living, and they were high-risk patients for conventional open AAA repair and were unsuitable candidates with a poor access route for EVAR.

\section{SURGICAL TECHNIQUES}

1. The ipsilateral patent common iliac artery proximally placed to the long complete occlusion of the external iliac artery was exposed through a retroperitoneal approach. Simultaneously, bilateral common femoral arteries were exposed by inguinal incision.

2. Percutaneous transluminal angioplasty (PTA) with stent placement was performed for short occlusion and/or stenosis of the contralateral external iliac artery antegradely and/or retrogradely.

3. An 8-mm Dacron graft was anastomosed in an end-to-side fashion to the ipsilateral distal common iliac artery as a safe iliac access conduit for EVAR (Figure 2 (a)).

4. After completion of the anastomosis, the delivery sheath (18-Fr introducer) of EVAR was inserted to the proximal site of the iliac conduit through a purse-string suture of the distal side aspect of the Dacron graft itself to avoid excess bleeding under fluoroscopic guidance.

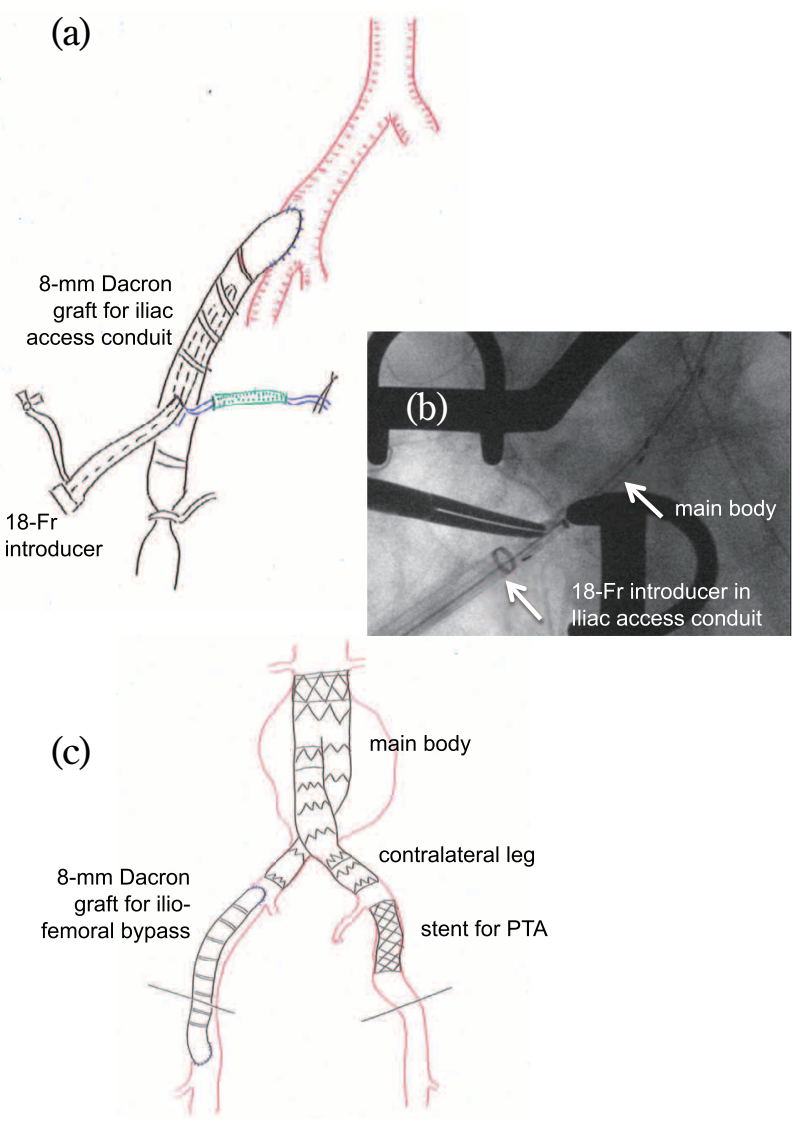

Figure 2. Operative schema and intraoperative fluoroscopic image for endovascular repair in Case 1. An 8-mm Dacron graft was anastomosed in an end-to-side fashion to the right distal common iliac artery as a safe iliac access conduit for endovascular aortic aneurysm repair (a). Main body of stent-graft was inserted through a delivery sheath (18-Fr introducer) at the distal side aspect of the iliac access conduit under intraoperative fluoroscopic guidance (b). Completion of endovascular aortic aneurysm repair, PTA and iliofemoral bypass was shown (c). Fr= French, PTA=percutaneous transluminal angioplasty 
5. The main body of Y-type stent-graft was inserted through the delivery sheath and deployed from below the inferior renal artery orifice to proximal site of the common iliac artery-conduit anastomosis (Figure 2 (b)).

6. An additional stent-graft for the contralateral leg was delivered in the contralateral common iliac artery through the external iliac artery immediately reconstructed in advance by PTA.

7. After completion of EVAR, the iliac access conduit was used for ipsilateral iliofemoral bypass graft under the inguinal ligament (Figure 2 (c)).

\section{POSTOPERATIVE COURSES}

Perioperative blood loss was well limited. Both postoperative courses were uneventful. There were no significant complications associated with EVAR, PTA and an iliofemoral bypass. Follow-up is complete in both patients and they are free from aggravation of heart and renal failure after operation. To date, both conduits remain patent, and both patients are alive and doing well with improved activities of daily living.

\section{DISCUSSION}

An iliac access conduit for endovascular access was first reported as "perspective" and "access problems" by Parodi et al. (2). They reported that an aorto-uni-iliac stent-graft combined with the extraanatomical femorofemoral bypass is useful for AAA with diseased ilio-femoral arteries. Subsequently, EVAR using the iliac access conduit and ipsilateral ilio-femoral bypass through a retroperitoneal approach was first reported by Yano et al. in 2001 (3) and by Lee WA et al. in 2002 (4). We modified this technique together with PTA for contralateral iliac arterial disease.

The techniques of EVAR using the iliac access conduit concomitant with PTA of contralateral iliac artery disease and ipsilateral iliofemoral bypass using the iliac access conduit were superior in terms of not only completion of an anatomical vascular reconstruction in patients with bilateral iliac artery occlusive diseases but also not putting the thick delivery sheath of the main body of EVAR through the native narrow and tortuous diseased iliac arteries, which potentially decreases the risk of access route injuries (Figure 3). (a)

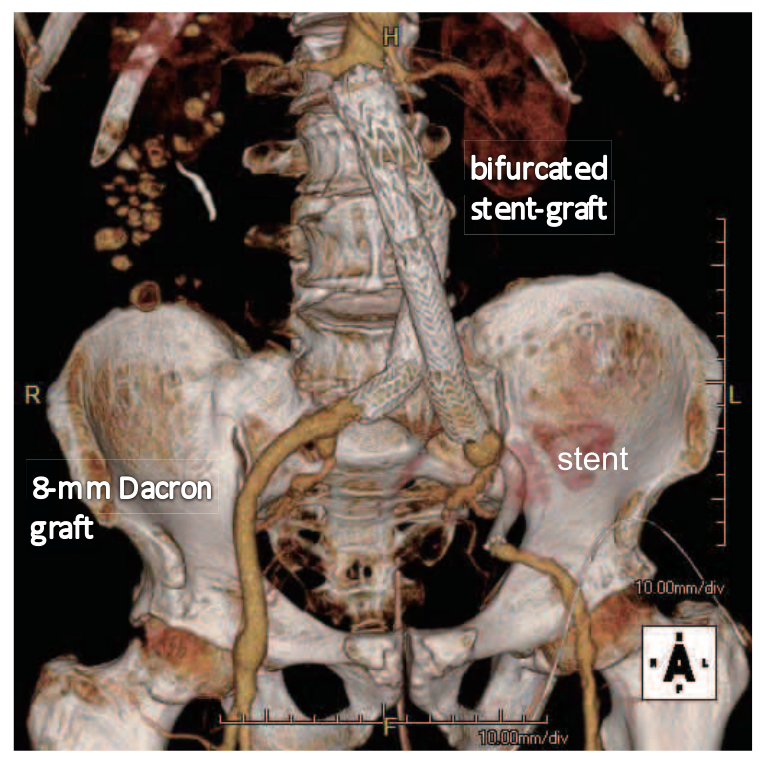

(b)

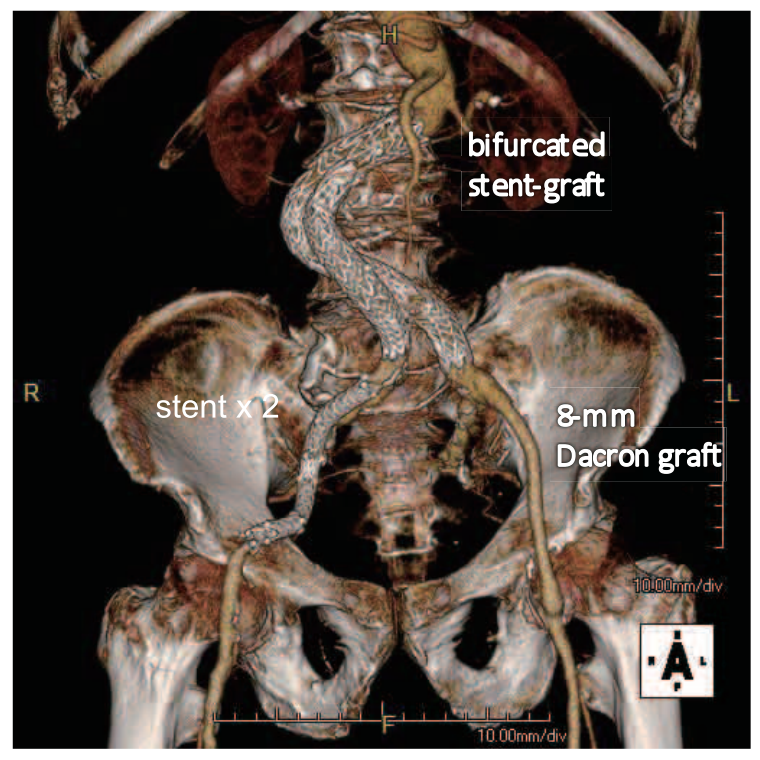

Figure 3. Postoperative 3D-computed tomo-angiography. Postoperative 3D-computed tomo-angiography showed successful completion of an anatomical vascular reconstruction in Case 1 (a) and in Case 2 (b).

We performed EVAR using an 18-French introducer not from the terminal end of the iliac access conduit but from the side aspect of the iliac access conduit. This contributed greatly to reduction of bleeding during passage of the delivery sheath (5).

We believe that the use of such techniques will ultimately increase the number of patients eligible for EVAR and avoid devastating access-related complications. 


\section{CONFLICT OF INTEREST}

We have no conflict of interests to disclose.

\section{REFERENCES}

1. Woodburn KR, Chant H, Davies JN, Blanshard KS, Travis SJ : Suitability for endovascular aneurysm repair in an unselected population. $\mathrm{Br} \mathrm{J}$ Surg $88: 77-81,2001$

2. Parodi JC, Barone A, Piraino R, Schonholz C : Endovascular treatment of abdominal aortic aneurysms. J Endovasc Surg 4 : 102-110, 1997
3. Yano OJ, Faries PL, Morrissey N, Teodorescu V, Hollier LH, Marin ML : Ancillary techniques to facilitate endovascular repair of aortic aneurysms. J Vasc surg 34 : 69-75, 2001

4. Lee WA, Berceli SA, Huber TS, Seeger JM : A technique for combined hypogastric artery bypass and endovascular repair of complex aortoiliac aneurysms. J Vasc Surg $35: 1289-91$, 2002

5. Abu-Ghaida AM, Clair DG, Greenberg RK, Srivastava S, O'Hara PJ, Ouriel K : Broadening the applicability of endovascular aneurysm repair : the use of iliac conduits. J Vasc Surg 36 : 111-117, 2002 